\title{
Design and Control of a Soft and Continuously Deformable 2D Robotic Manipulation System*
}

\author{
Andrew D. Marchese ${ }^{1}$, Konrad Komorowski ${ }^{1}$, Cagdas D. Onal ${ }^{2}$, and Daniela Rus ${ }^{1}$
}

\begin{abstract}
In this paper we describe the design, fabrication, control, and experimental validation of a soft and highly compliant 2D manipulator. The arm consists of several body segments actuated using bi-directional fluidic elastomer actuators and is fabricated using a novel composite molding process. We use a cascaded PI and PID computation and novel fluidic drive cylinders to provide closed-loop control of curvature for each soft and highly compliant body segment. Furthermore, we develop algorithms to compute the arm's forward and inverse kinematics in a manner consistent with piece-wise constant curvature continuum manipulators. These computation and control systems enable this highly compliant robot to autonomously follow trajectories. Experimental results with a robot consisting of six segments show that controlled movement of a soft and highly compliant manipulator is feasible.
\end{abstract}

\section{INTRODUCTION}

Traditional rigid industrial robots provide optimal solutions for factory automation, where fast, precise, and controllable motions are utilized for repetitive tasks in structured environments. However, in natural environments and human-centric operations where safety and adaptability to uncertainty are fundamental requirements, soft robots may serve as a better alternative to automation. Soft robots are designed with a continuously deformable backbone providing theoretically infinite degrees of freedom; see review by Trivedi, Rahn, Kier, and Walker [1]. Soft robots can conform to variable but sensitive environments exemplified by Chen et al. [2]. They can adaptively manipulate and grasp unknown objects varying in size and shape [3]. And their high dexterity allows them to squeeze through confined spaces [4]. However, a fundamental limitation in designing robots to be softer and more compliant is that the robots become increasingly unconstrained, making predictable and controlled movement difficult. Typically there is a balance between compliance and internal kinematic constraints that make controlled movement feasible.

In this work we demonstrate precise closed-loop positional control for a highly compliant planar continuum manipulator made almost entirely of soft silicone rubber. The arm has the advantage of being more compliant than most soft continuum manipulators, e.g. a force of only $1.5 \mathrm{~N}$ is required to hold

\footnotetext{
*This work was supported by the National Science Foundation, grant numbers NSF IIS1226883 and NSF CCF1138967 as well as the National Science Foundation Graduate Research Fellowship Program, Primary Award 1122374

${ }^{1}$ A. D. Marchese, K. Komorowski, and D. Rus are with the Computer Science and Artificial Intelligence Laboratory, Massachusetts Institute of Technology, Cambridge, MA 02139, USA \{andy, kkom, rus\}ecsail.mit.edu

${ }^{2}$ C. D. Onal is with the Department of Mechanical Engineering, Worcester Polytechnic Institute, Worcester, MA 01609, USA cdonal@wpi . edu
}

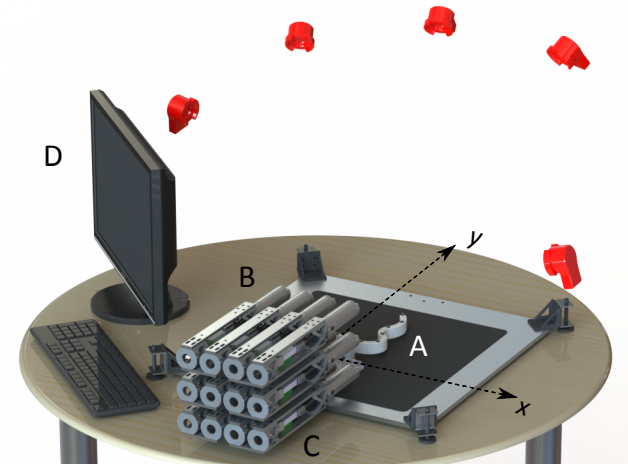

Fig. 1. An overview of the major components comprising the soft robotic manipulation system. These are: a soft and highly compliant manipulator (A), fluidic drive cylinder array (B), multi-segment kinematics computation (D), and arm segment curvature controllers (C). External cameras are shown in red.

the tip of the $23 \mathrm{~cm}$ long manipulator to it's base in a loop shape. The positioning capability is enabled by innovation in both design and computation that provides stable real-time curvature control of the manipulator's soft pneumatic body segments despite their high compliance and lack of kinematic constraints.

Here we solve the previously unaddressed problem of controlling arc space parameters for an entirely soft and highly compliant pneumatic arm. In the planar inextensible case, this means controlling body segment curvatures. Each of the arm's serially connected segments are composed of fluidic elastomer actuators (FEAs) [5] and these actuators deform into curvature about a central axis when pressurized [6]. Accordingly, we make a simplifying piece-wise constant curvature (PCC) assumption to model the forward and inverse kinematic relationship between the arm's arc space (i.e., segment curvatures and lengths) and task space (i.e., end effector pose) in a manner consistent with traditional continuum manipulation literature, reviewed by Webster and Jones [7]. This assumption means each body segment of a multi-segment arm is assumed to deform with constant curvature. An early use of PCC appears in Hannan and Walker's work with a bending robotic trunk [8]. We use forward and inverse kinematics algorithms to solve for arc space set-points for our feedback controller in real-time. This controller uses a cascaded PI and PID computation as well as fluidic drive cylinders to control the curvature of the soft and highly compliant arm segments, allowing for precise position control of the manipulator.

In general, the design of existing soft position controlled 
manipulators are not very soft. Originally, many hard hyper redundant and hard continuum robots [8] [9] [10] used an array of servomotors or linear actuators to pull cables that moved rigid connecting plates located between body segments. Some soft robots have adopted a similar actuation scheme consisting of tendons pulling rigid fixtures embedded on a continuously deformable backbone as seen in the soft manipulators controlled by Gravagne and Walker [11], McMahan, Jones, and Walker [12], and Camarillo, Carlson, and Salisbury [13]. There is an example of a soft rubber position controlled arm using cables without rigid plates by Wang et al. [14], but the arm consists of only one actuated segment and therefore does not require internal fixtures. Another common design of position controlled soft manipulators involves distributed pneumatic muscle actuators (PMAs). Here, PMAs are embedded throughout the robot's body. Notable examples include OctArm IV [3] which uses 18 air muscle actuators distributed throughout 4 arm segments, the continuum manipulator developed by Pritts and Rahn [15] which uses 14 McKibben actuators within two body segments, and the manipulator developed by Kang, Branson, Zheng, Guglielmino, and Caldwell [16] which uses 24 PMAs within 6 body segments. Again, these designs are not entirely soft but rather include rigid plates between segments for actuator mounting and kinematic constraint.

To the best of our knowledge, highly compliant robots whose bodies are made from soft rubber and distributed pneumatic actuators are not capable of closed-loop curvature control. Prior works in this field use open-loop control, but this approach is not sufficient for providing accurate control of body segment curvature. Most fluid powered soft robots use open-loop valve sequencing (i.e., a valve is turned on for a duration of time to pressurize the actuator and then off to either hold or deflate it) to control body segment bending. For instance, there are soft rolling robots [5] [17] [18] made of FEAs that use this control approach. Also a soft snake-like robot developed by Onal and Rus [19] uses this open-loop scheme to control 8 distributed FEAs among 4 body segments to enable serpentine locomotion. Again, Shepherd et al. use an open-loop valve controller to drive body segment bending in an entirely soft multigait robot [4] and then passive control in an explosive, jumping robot [20]. Martinez et al. [21] develop manually operated elastomer tentacles containing 9 PneuNet actuators embedded within 3 body segments. There is also an example of controlling a soft pneumatic inchworm-like robot using servo-controlled pressure described in [22]. Here, a PWM approach is used to drive rapid valve switching to continuously vary airflow.

Open-loop control is also common for soft rubber robots that do not use pneumatic actuation. For example, previous work on soft bioinspired octopus-like arms developed by Calisti et al. [23] demonstrate open-loop capabilities like grasping and locomotion [24] [25]. Umedachi, Vikas, and Trimmer [26] developed a soft crawling robot that uses an open-loop SMA driver to control body bending.

Our work differs from previous literature in both design and control. At a low level, we use a pair of position- controlled linear fluidic drive cylinders to independently pressurize the robot's body actuators. This arrangement is superior to solenoid valves since it enables precise analog control of airflow into and out of body actuators. At a higher level, the feedback system controls the curvature of each of the robot's body segments according to the arm's modeled kinematics. This allows the system to autonomously control the pose of points along the arm. The contributions of this paper include:

- The design of a soft and highly compliant planar multisegment manipulator

- The design of a novel drive system for soft fluidic robots, the fluidic drive cylinder

- Closed-loop curvature control for a soft and highly compliant planar pneumatic robot

- Autonomous position control of points along a multisegment soft and highly compliant continuum arm.

\section{DESIGN}

The soft robotic manipulation system is composed of several subsystems. Figure 1 depicts the aggregate system, while highlighting the major subsystems: a soft and highly compliant manipulator (A), fluidic drive cylinder array (B), multi-segment kinematics computation (D), arm segment curvature controllers (C).

\section{A. Soft Arm}

The soft and continuously deformable arm (Fig. 1A) functions as a manipulator and interacts with the environment. In this work the manipulator's reachable envelope is constrained to the X-Y plane illustrated in Fig. 1. By volume, over 97 percent of the arm is composed of soft silicone rubber, excluding the feet. Structurally, the arm is composed of serially connected homogeneous elastomeric bending segments. An individual segment is detailed in Fig. 2. Each segment is fundamentally a fluidic elastomer actuator [5] [17] and capable of bending bi-directionally in the X-Y plane. Bending is the result of expansion and contraction of agonistic and antagonistic fluidic channel groupings (a and b) embedded within the segment's elastomer (c). Channel deformation is generated by pressurizing or vacuuming internal fluid (d), which induces stress in the elastomer. An inextensible but continuously deformable constraining film (e) separates agonistic and antagonistic fluidic channels. The constraint serves to transform channel deformation into segment curvature by providing a neutral axis around which the segment bends. Fig. 2B depicts a segment in a state of bending. Elongation of the agonistic channel grouping under pressurization (red) causes the inextensible constraint to assume negative curvature. Contraction of the antagonistic channel grouping under vacuum (cyan) allows for increased bending, but is not required. Elastomer between channels promotes channel deformation along the neutral axis and reduces extension along $\vec{r}$, orthogonal to the neutral axis.

The soft arm, see Fig. 3, can be composed of any number of segments (A). Markers are located at the interface between 


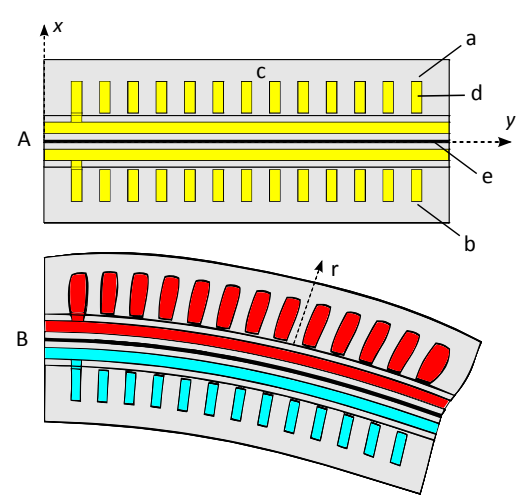

Fig. 2. Cross section view of a soft arm segment depicting both a relaxed (A) and bent (B) state. Here, agonistic (a) and antagonistic (b) channel groupings are embedded within silicone elastomer (c) and separated by an inextensible constraining layer (e). These channels house fluid (d) (yellow) which can be either pressurized (red) or vacuumed (cyan).

segments (D), making these points identifiable. The starting point of the arm's first segment (B) is grounded to the platform on which the arm moves. Ball transfers (C) are also located at segment endpoints and enable the arm to move in the two-dimensional plane with minimal friction. In many experiments conducted throughout this work the arm's end effector (E) is controlled.

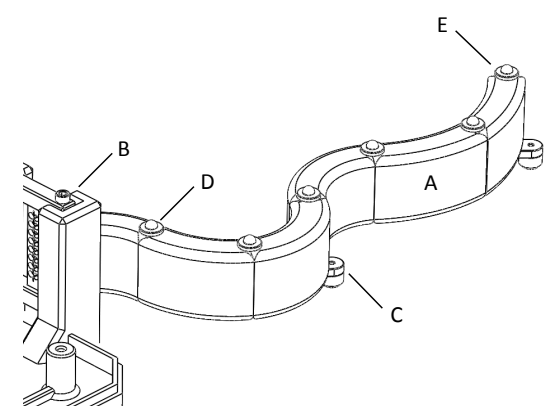

Fig. 3. The soft arm is composed of homogeneous and independently actuated segments (A). The base of the arm's first segment is fixed and the end of its last segment is the end effector (E). Markers (D) identify the endpoints of each segment and ball transfers (C) help the arm move with minimal friction.

\section{B. Fluidic Drive Cylinders}

In order to independently actuate arm segments, an array of custom fluidic drive cylinders (Fig. 1B) were developed. These cylinders produce volumetric changes within the above mentioned embedded fluidic channels. Electric linear actuators are directly coupled to and control the positional displacement of pistons within the fluidic cylinders. Accordingly, these linear actuators govern the volumetric displacement of fluid out of the cylinders and into the embedded channels within the elastomeric arm segments, and vice versa.

An open loop, linear time-invariant dynamic model was created to approximate the performance of the fluidic drive cylinder. Although the model is not used in the control of the cylinder because of its simplifications (in reality many of these parameters are nonlinear), it served to identify the impact design decisions have on input-output relationships. Fig. 4 shows a simplified schematic representation of the system and the parameters considered in developing the model.

The equations of motion for the drive cylinder are written below. The piston's linear motion is described as:

$$
F_{a}-P_{2} A_{p}=m_{p} \ddot{x_{2}}
$$

Where $F_{a}$ is the force exerted by the linear actuator on the piston, $P_{2}$ is the fluid pressure insider the cylinder, $A_{p}$ and $m_{p}$ are the cross sectional area and the mass of the piston. Lastly, $x_{2}$ is the piston displacement. The volumetric fluid flow into the elastomeric channels is approximated as:

$$
\frac{P_{2}-P_{1}}{R_{t}}=\left(C_{a}+C_{c}\right) \dot{P}_{1}
$$

Here, $P_{1}$ is the fluid pressure within the channels of the arm segment. $R_{t}$ is the resistance of the connecting tube. $C_{a}$ and $C_{c}$ are the compliances of the elastomeric channels and fluid respectively. The volumetric fluid flow into the cylinder is approximated as:

$$
A_{p} \dot{x_{2}}+\frac{P_{1}-P_{2}}{R_{t}}=C_{c} \dot{P_{2}}
$$

And lastly the force output of the linear actuator can be approximated as:

$$
F_{a}=-\frac{\gamma p}{R_{m}} \dot{x_{2}}+\frac{\gamma}{R_{m}} e_{i n}
$$

Above, $\gamma$ is a parameter relating force and motor current in the linear actuator and $p$ relates counter emf voltage to linear velocity. $R_{m}$ is the motor's resistance, and $e_{i n}$ is the input motor voltage.

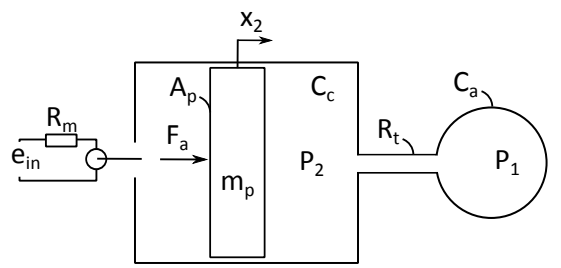

Fig. 4. Parameters used in developing a simplified model of a fluidic drive cylinder. At the left is a schematic representation of the electric linear actuator, at the middle is a representation of the piston and cylinder, and at the right is a representation of an elastomeric channel grouping within an arm segment.

Using the aforementioned equations of motion, the open loop LTI system model can be written as Eqn. (5). Combining Eqn. (1) and (4) yields the first row. The second row is Eqn. (2), and the third row is Eqn. (3). Approximations of the model's parameters are listed in Table I.

$$
\left[\begin{array}{l}
\ddot{x}_{2} \\
\dot{p}_{1} \\
\dot{p}_{2}
\end{array}\right]=\left[\begin{array}{ccc}
-\frac{\gamma p}{m_{p} R_{m}} & 0 & -\frac{A_{p}}{m_{p}} \\
0 & -\frac{1}{\left(C_{c}+C_{a}\right) R_{t}} & \frac{1}{\left(C_{c}+C_{a}\right) R_{t}} \\
\frac{A_{p}}{C_{c}} & \frac{1}{C_{c} R_{t}} & -\frac{1}{C_{c} R_{t}}
\end{array}\right]\left[\begin{array}{l}
\dot{x}_{2} \\
p_{1} \\
p_{2}
\end{array}\right]+\left[\begin{array}{c}
\frac{\gamma}{m_{p} R_{m}} \\
0 \\
0
\end{array}\right] e_{i n}
$$

Two drive cylinders are used to control a single bidirectional segment. Although the mapping of either the 
TABLE I

APPROXIMATIONS OF FLUIDIC DRIVE CYLINDER PARAMETERS

\begin{tabular}{cccccccc}
\hline \hline$g$ & $p$ & $m_{p}$ & $R_{m}$ & $A_{p}$ & $C_{c}$ & $C_{a}$ & $R_{t}$ \\
125 & 327 & 0.19 & 17 & 7.9 & $2.1 \mathrm{e}-10$ & $2.0 \mathrm{e}-9$ & $1.7 \mathrm{e} 8$ \\
$\mathrm{~N}$ & $\frac{\mathrm{V} s}{\mathrm{~m}}$ & $\mathrm{~kg}$ & $\Omega$ & $\mathrm{cm}^{2}$ & $\frac{\mathrm{m}^{3}}{\mathrm{~Pa}}$ & $\frac{\mathrm{m}^{3}}{\mathrm{~Pa}}$ & $\frac{\mathrm{Pa} \mathrm{s}}{\mathrm{m}^{3}}$ \\
\hline
\end{tabular}

agonistic or antagonistic channel group deformation is monotonically related to a single piston's displacement, when considering bi-directional segment movement as well as positive and negative curvatures, the two drive cylinders must be controlled synchronously. One piston is held still and the other piston is moved in either forward or reverse to increase or decrease curvature. There are four distinct states of the fluidic drive cylinders as detailed in Fig. 5.
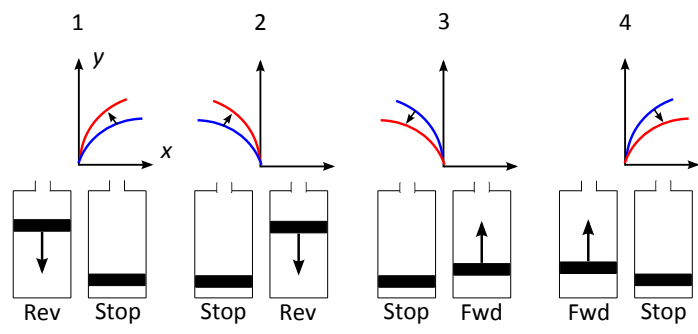

Fig. 5. Diagram depicting the four driving states of the two fluidic drive cylinders used to control an arm segment. These states depend on the error in curvature (measured (blue) - target (red) arcs) as well as the sign of the curvature (right hand rule). (1) The curvature is negative and the error is positive. (2) The curvature is positive and the error is negative. (3) The curvature is positive and the error is positive. (4) The curvature is negative and the error is negative.

\section{FABRICATION}

\section{A. Soft Arm}

In this work we fabricated an arm from various soft and semi-soft materials using the processes described in the following. Fig. 6 details the fabricated components of the arm. Table III contains the superscript references to machine tools and materials. The arm is composed of six segments. Seven constraint supports (d) were printed using a $3 \mathrm{D}$ printing machine ${ }^{1}$ and placed into a constraint layer mold (f), which was also printed. The constraint film (c) was then cut from $0.25 \mathrm{~mm}$ ABS plastic film using a laser ${ }^{2}$ and inserted through the aforementioned supports. Above and below the constraint film, eight pieces of silicone tubing (a) were also threaded through the supports. Silicone rubber ${ }^{3}$ was then mixed and poured into the constraint layer mold, immersing tubing, film, and supports in a layer of elastomer to create the composite constraint layer $(\mathrm{g})$. The silicone was immediately degassed using a vacuum chamber ${ }^{4}$ before curing.

Once cured, small holes were created in the constraint layer to pierce the embedded tubing at specific locations allowing each line to independently address a group of fluidic channels. Elastomer pieces containing channels (b) were casted and cured separately using a similar molding technique. Two channel pieces (b) were carefully attached to both faces of the constraint layer using a thin layer of silicone. Lastly, the printed feet (e) were attached to the constraint supports to create an attachment point for ball transfers, see also Fig. 3C. Two types of feet were used. Four feet hold a single ball transfer, whereas two feet hold two ball transfers. These mechanisms prevent the arm from tipping and help constrain the arm's motion to the X-Y plane. Table II lists physical arm properties.

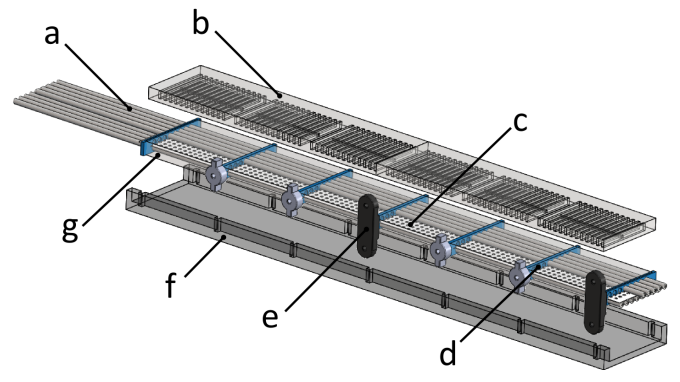

Fig. 6. Fabrication details of the soft arm: silicone tubing (a), elastomer pieces containing channels (b), constraint film (c), constraint supports (d), feet (e), constraint layer mold (f), and composite constraint layer (g).

TABLE II

PHYSICAL ARM PROPERTIES

\begin{tabular}{lrlr}
\hline \hline Parameter & Value & Parameter & Value \\
\hline channel height & $3 \mathrm{~mm}$ & elastomer height (b) & $5 \mathrm{~mm}$ \\
constraint height $(\mathrm{g})$ & $6 \mathrm{~mm}$ & total segment height & $19 \mathrm{~mm}$ \\
channel length & $1 \mathrm{~mm}$ & segment length & $33 \mathrm{~mm}$ \\
arm length & $198 \mathrm{~mm}$ & channel thickness & $25.4 \mathrm{~mm}$ \\
segment thickness & $31.8 \mathrm{~mm}$ & arm thickness & $47 \mathrm{~mm}$ \\
arm weight & $162 \mathrm{~g}$ & tubing I.D. & $1.0 \mathrm{~mm}$ \\
tubing O.D. & $2.2 \mathrm{~mm}$ & & \\
\hline
\end{tabular}

\section{B. Fluidic Drive Cylinders}

Fluidic drive cylinders are mechanisms which interface the computational and algorithmic aspects of the manipulation system with the soft arm. Specifically, they input digital command signals from a control algorithm and generate fluidic pressure that drives the curvature of the arm segments. Fundamentally, these operate by using electrical energy to displace a piston within a fluidic cylinder.

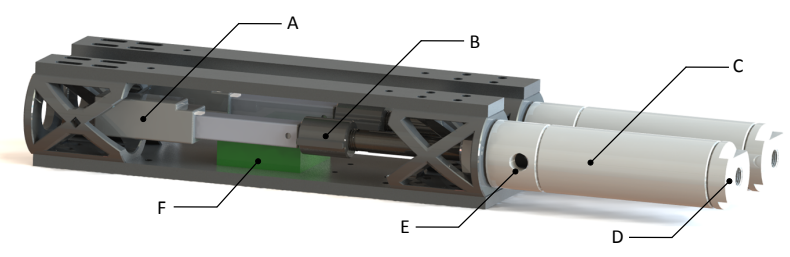

Fig. 7. Overview of two fluidic drive cylinders used to drive the curvature of a bi-directional arm segment. An electric linear actuator (A) is directly coupled (B) to the piston of a fluidic cylinder (C). Fluid is displaced through the inlet (E) and outlet (D) of the cylinder. A motor controller (F) allows digital command signals to govern fluid movement.

Fig. 7 illustrates the components of a fluidic drive cylinder. An electric linear actuator ${ }^{5}$ (A) is directly coupled to the 
piston of a fluidic cylinder ${ }^{6}$ (C) via a threaded coupler (B). The outlet of the cylinder (D) is connected to a single tube. The tube then connects to a channel grouping within the arm segment. The inlet (E) is open to ambient air. A motor controller $^{7}(\mathrm{~F})$ inputs digital commands from a controller and outputs drive signals to the linear actuator.

TABLE III

COMMERCIALLY AVAILABLE TOOLS AND EQUIPMENT

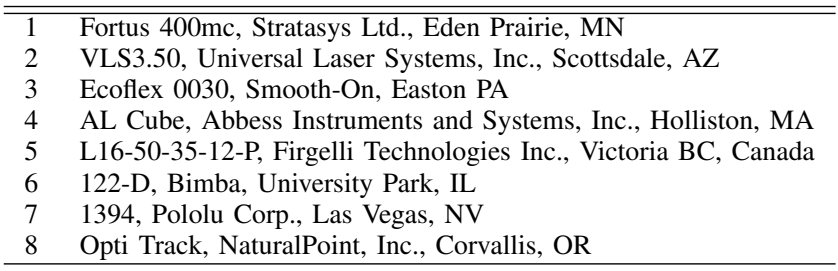

\section{CONTROLS}

\section{A. Curvature Estimation}

In order to control the pose of arbitrary points along the soft robot arm in task space, it is first necessary to estimate an arm segment's state in arc space using available localization data. Based on previous results describing the deformation of a fluidic elastomer actuator [17], we assume the state of an arm segment can be represented by a signed curvature $k$ and knowledge of its starting orientation, $\theta_{0}$. This PCC assumption is common in continuum manipulation [8] [7]. The available data to estimate this state is the $\mathrm{X}-\mathrm{Y}$ position of each body segment's start and end point markers. Therefore we develop a single segment inverse kinematics transformation (i.e., from task space to arc space). Fig. 8 visualizes the algorithm we used for determining $k$ given a body segment's start point $A$ and end point $B$ as well as $\theta_{0}$. We refer to this as the curvature algorithm (Algorithm 1). Although we provide our approach, the problem of relating an initial angle and the positions of endpoints to curvature has been previously addressed [8][27].

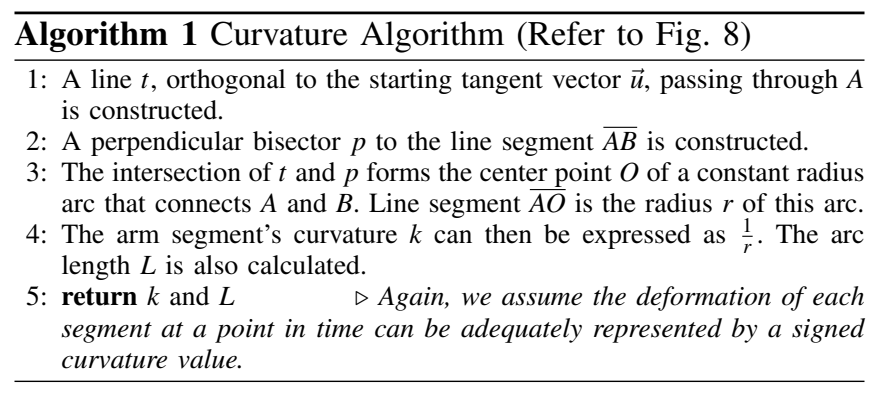

\section{B. Forward Kinematics}

Knowing independent segment curvatures allows us to write the forward kinematics of serially connected bidirectional bending segments, an approach developed by [19]. The orientation at any point $s \in\left[0, L_{i}\right]$ along the arc representing segment $i$ within a chain of $n$ segments composing the arm can be expressed as:

$$
\theta_{i}(s)=k_{i} s+\theta_{i}(0)
$$

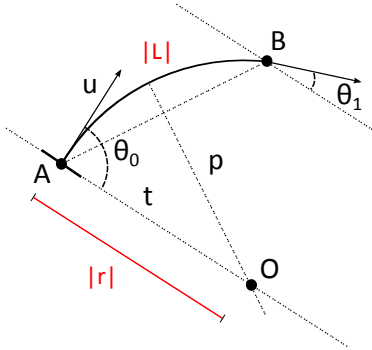

Fig. 8. Visualization of the algorithm used to determine the state of a soft arm segment at a given point in time. The segment's start point, $A$, and end point, $B$, are measured and the initial orientation, $\theta_{0}$, of the segment is provided. Segment curvature, $k$, is determined.

Because these segments are serially connected and continuous we assume $\theta_{i}(0)=\theta_{i-1}\left(L_{i-1}\right)$. In the case of our manipulator, this allows the forward kinematics algorithm (Algorithm 2) to uniquely identify the state of the entire arm by starting at the grounded base segment $(i=0)$ where the orientation is a priori information. Subsequently, each consecutive segment's state is determined. as:

The position of any point along the arm can be expressed

$$
\begin{aligned}
& x_{i}(s)=x_{i-1}\left(L_{i-1}\right)+\int_{0}^{s} \cos \left[\theta_{i}\left(s^{\prime}\right)\right] \mathrm{d} s^{\prime} \\
& y_{i}(s)=y_{i-1}\left(L_{i-1}\right)+\int_{0}^{s} \sin \left[\theta_{i}\left(s^{\prime}\right)\right] \mathrm{d} s^{\prime}
\end{aligned}
$$

Frequently throughout this work we will refer to the manipulator's end effector, which is defined as $\vec{w}_{6}=\left[x_{6}\left(L_{6}\right)\right.$, $\left.y_{6}\left(L_{6}\right), \theta_{6}\left(L_{6}\right)\right]$. The forward kinematics algorithm (Algorithm 2) for recursively determining a point on the arm located at $s$ on segment $i$ given curvature and length is provided.

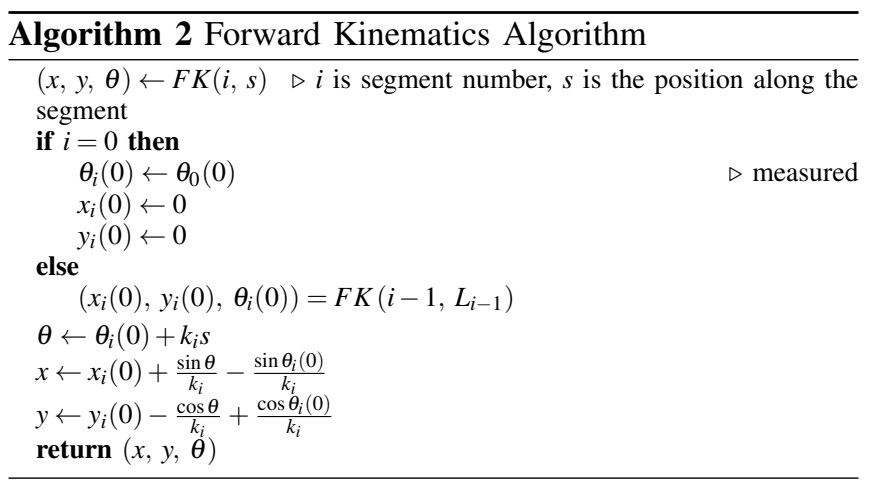

\section{Inverse Kinematics Algorithm}

Besides the curvature and forward kinematics algorithms (Algorithms 1 and 2 respectively), a critical component to the main control algorithm is the manipulation system's inverse kinematics algorithm (Algorithm 3), or IK algorithm. An iterative jacobian transpose approach is used [28][29]. For this work, it means we determine a small update to segment curvatures that will move a controlled point or points towards their desired pose, $\vec{w}_{d}=\left[x_{d}, y_{d}, \theta_{d}\right]$. Each iteration, the algorithm calculates the incremental curvature updates $\Delta k$. Upon completion, the curvatures required to 
attain the desired arm pose at that control time step are returned. In other words, given the start and end points of each arm segment and $\theta_{0}(0)$ as well as the desired pose(s), the algorithm determines a curvature discrepancy for each arm segment.

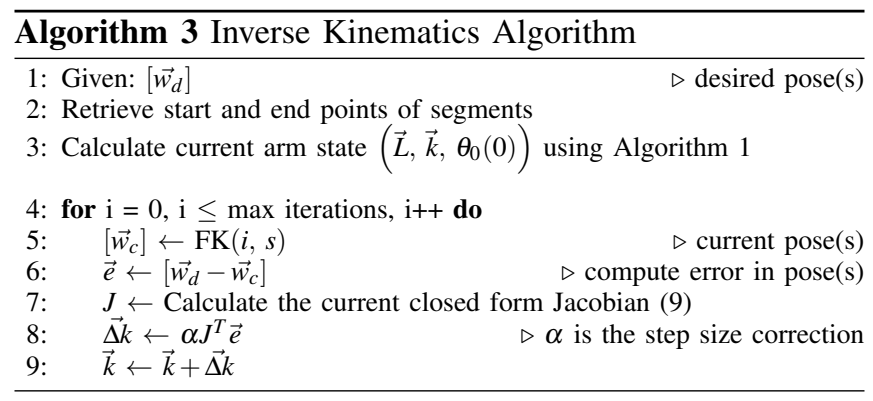

We are able to write the jacobian in closed-form and this is fundamental to the inverse kinematics algorithm (Algorithm $3)$.

$$
J=\frac{\partial \vec{w}\left(\vec{L}, \vec{k}, \theta_{0}(0)\right)}{\partial \vec{k}}
$$

A current arm state can be easily substituted into 9 allowing the IK algorithm to be run each iteration of the real-time controller.

\section{Main Control Algorithm}

The main control algorithm determines adjustments to segment curvatures in real-time that are required to move a point or points along the arm through their requested pose trajectory. Before using the inverse kinematics algorithm (Algorithm 3), the main controller ensures the integrity of measured data by comparing it to historical data. Once the required curvature updates are computed, this algorithm passes the information to the lower level segment controllers.

\section{E. Arm Segment Controller}

In order to drive arm segment curvatures to their required values, a closed-loop arm segment control algorithm was developed. This low-level control algorithm periodically receives discrepancies between the soft arm's measured and requested curvatures and uses a cascaded control structure to effectively adjust fluidic drive cylinders and resolve the error. The controller achieves this by running a PI computation on the curvature error in order to generate a new set-point for the positional control of the linear actuator. Due to the limitations of the used localization system $^{8}$, this outer loop runs at a relatively slow rate $(20 \mathrm{hz})$ and is initiated when the main control algorithm (Section IV D) produces a curvature error. The inner loop, or positional PID controller runs at $1 \mathrm{kHz}$ to bring the cylinder's piston displacement to the newly determined set-point. The cylinder's piston displacement is the primary manipulated variable as fluid pressure is monotonically related to segment curvature. Fig. 9 visualizes the cascaded control algorithm. Each of the arm's segments has its own controller.

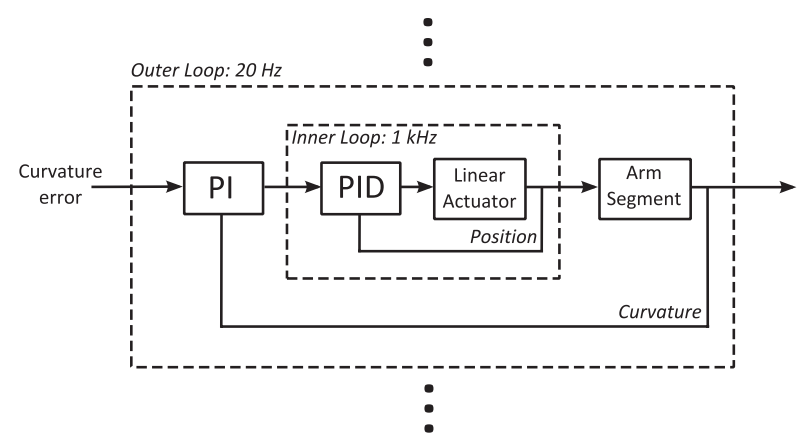

Fig. 9. Cascaded control feedback algorithm used to set the soft arm's curvatures. Each arm segment is controlled at a low-level by this nested curvature and positional controller. The inner loop runs substantial faster than the outer loop providing stable control over piston position.

\section{EXPERIMENTS}

The robotic manipulation system is able to accurately and precisely control the pose at points along the soft and continuously deformable arm in real-time. Specifically, the manipulation system can move the soft arm's end effector to a user specified pose. We refer to this capability as pointto-point movement. The arm's end effector can also track trajectories. Requested paths can be provided to the system in real-time. We refer to this capability as path tracking. In this work we show how we are able to achieve these fundamental capabilities while maintaining the most significant characteristic of this manipulation system, softness.

\section{A. Single Segment Curvature Tracking}

A fundamental result required for point-to-point movement and path tracking is the capability for an individual segment to track a curvature profile varying over time. Fig. 10 details both the target and measured curvature over time as well as the error of the arm's second segment. Here, the target profile is a sine wave of amplitude $5 \frac{1}{\mathrm{~m}}$ with a period of 9 seconds, centered about $-15 \frac{1}{\mathrm{~m}}$. A challenge for the controller is transitioning from driving either of the two fluidic cylinders to the other and this occurs when the segment's curvature passes zero. Fig. 11 details curvature tracking of the arm's fourth segment when a similar target sinusoidal profile is centered about $0 \frac{1}{\mathrm{~m}}$.

\section{B. Point-to-Point Movements}

In order to verify the system's ability to accurately and precisely control the pose of a point on the soft arm, pointto-point movement experiments were conducted. During the experiment, the manipulator's end effector was commanded to move to four reachable poses (see Fig. 12A-D). Before moving to one of the commanded poses, the arm was initialized to a resting state where all arm segments were depressurized. The time history of each marker position as well as the arm's state $\left(\vec{k}, \vec{L}, \theta_{0}(0)\right)$, as determined by the curvature and forward kinematics algorithms, were logged. The arm was moved to each target pose ten consecutive times. After a settling period, the end effector's error in pose was measured. The mean and standard deviation of the 

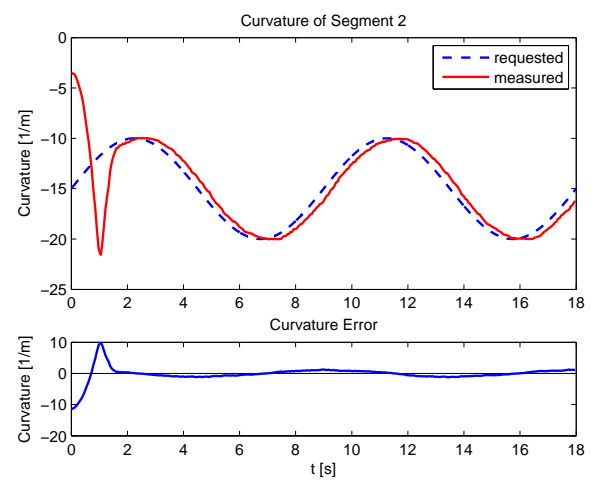

Fig. 10. At the top is the requested (blue dotted) and measured (red) curvatures over time for an individual arm segment. The sinusoidal trajectory is entirely negative, meaning only one drive cylinder is actuated. At the bottom the error in curvature (requested - measured) is shown.
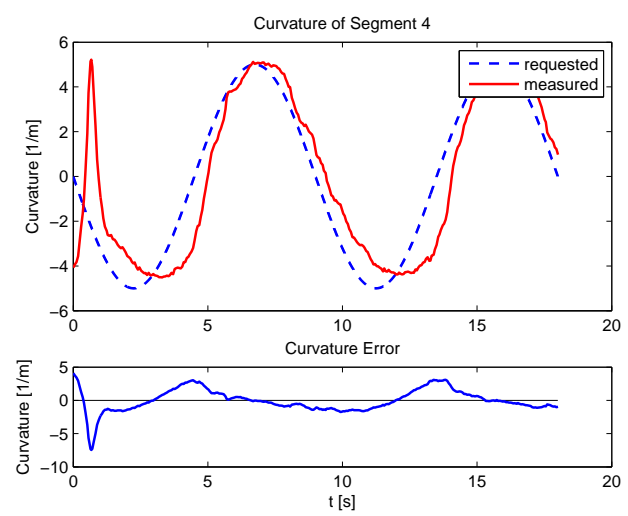

Fig. 11. Requested (blue dotted) and measured (red) curvatures over time for an individual arm segment. The sinusoidal trajectory is centered about zero, meaning both drive cylinders are actuated. Error in curvature (requested - measured) is shown.

positional and rotational error over all ten trials are reported for the four target poses in Table IV. Fig. 12 visualizes the arm's pose for each trial as well as the target poses.

TABLE IV

MEAN ERRORS AND S.D. FOR POINT-TO-POINT MOVEMENTS.

\begin{tabular}{ccc}
\hline \hline Point & Mean \pm S.D. Positional Error $(\mathrm{cm})$ & Rotational Error $(\mathrm{deg})$ \\
\hline A & $0.95 \pm 0.42$ & $1.75 \pm 1.26$ \\
B & $0.45 \pm 0.17$ & $0.58 \pm 0.58$ \\
C & $0.61 \pm 0.13$ & $0.91 \pm 0.58$ \\
D & $0.84 \pm 0.22$ & $1.28 \pm 0.60$ \\
\hline
\end{tabular}

\section{Path Tracking}

In order to verify the system's ability to accurately and precisely follow a trajectory in real-time, path tracking experiments were conducted. Again, the robot's end effector was controlled. Target poses were updated every iteration of the central control algorithm. Before each experimental trial the arm was initialized to a resting state where all arm segments were depressurized. During the experiment, the same data as in point-to-point experiments were collected.

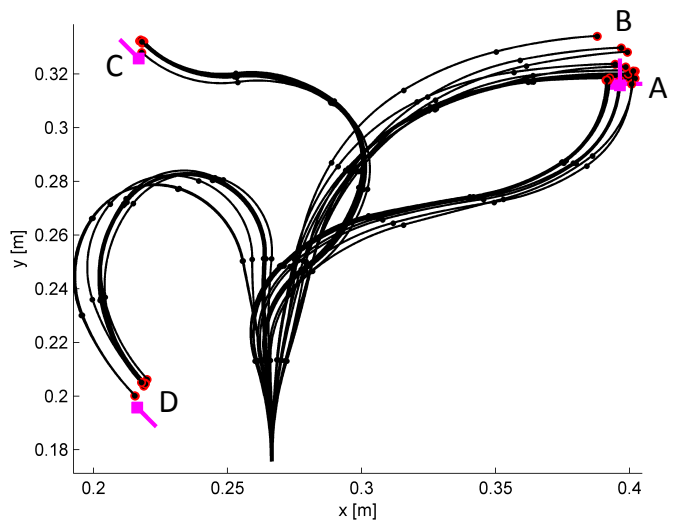

Fig. 12. Point-to-point movement results. The magenta squares and lines represent the end effector's four target poses (A-D). The red circles and black curves represent the arm's measured end effector positions, curvatures, and segment endpoints for each independent trial.

A total of ten trials where conducted. Fig. 13 details the Lshaped path the arm was commanded to follow (magenta). The arm's measured curvature (black) at the experiment's start ( $\mathrm{t}=0)$, the path's start (A), and path's end (B) is shown for an exemplary trial. The end effector's measured pose is shown at each time step along the path. Fig. 14 shows the compiled results for all ten trials. The mean positional and rotational error (red line) is calculated at each moment in time and overlayed on individual trial errors (blue line).

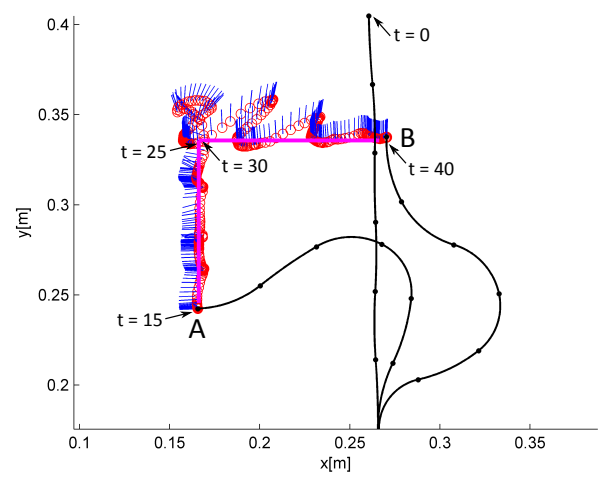

Fig. 13. An exemplary path tracking experimental trial. The L-shaped target path of the arm is shown in magenta. The vertical line has a target orientation of $\pi$ and the horizontal line has a target orientation of $\frac{\pi}{2}$. The arm's measured curvature at the path's start (A) and end (B) is shown (black). The end effector's measured pose is shown at each time step. End effector orientation is shown in blue and position is represented as red circles. Important path times are noted.

\section{CONCLUSIONS}

This paper outlined an approach to designing and controlling a pressure-operated soft robotic manipulator. The developed forward and inverse kinematic models were presented and we showed how they integrate into an autonomous control system for the robot. Finally, an arm consisting of six independently controllable segments was analyzed on its single section curvature tracking, point-to-point movement 

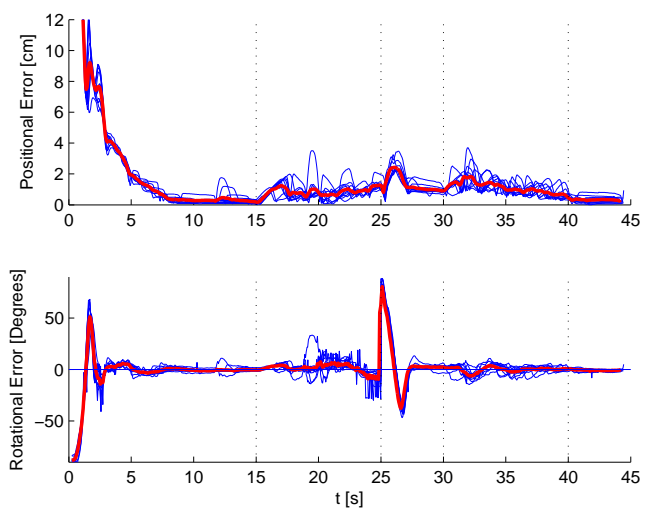

Fig. 14. Line tracking results from all ten trials. The positional and rotational error of the arm's end effector are reported as a function of time. Mean error (red) is calculated at each moment in time and overlayed on individual trial errors (blue). Vertical lines represent important timing events, see Fig. 13

accuracy, and path tracking accuracy. We demonstrate that we can control a soft and highly compliant 2D manipulator with a point-to-point mean positional accuracy of $0.71 \mathrm{~cm}$ and mean rotational accuracy of $1.1^{\circ}$.

\section{ACKNOWLEDGMENT}

A special thanks to Robert Katzschmann from the Distributed Robotics Laboratory for his extensive feedback.

\section{REFERENCES}

[1] D. Trivedi, C. D. Rahn, W. M. Kier, and I. D. Walker, "Soft robotics: Biological inspiration, state of the art, and future research," Applied Bionics and Biomechanics, vol. 5, no. 3, pp. 99-117, 2008.

[2] G. Chen, M. T. Pham, and T. Redarce, "Development and kinematic analysis of a silicone-rubber bending tip for colonoscopy," in Intelligent Robots and Systems, 2006 IEEE/RSJ International Conference on, 2006, pp. 168-173.

[3] W. McMahan, V. Chitrakaran, M. Csencsits, D. Dawson, I. D. Walker, B. A. Jones, M. Pritts, D. Dienno, M. Grissom, and C. D. Rahn, "Field trials and testing of the octarm continuum manipulator," in Robotics and Automation, 2006. ICRA 2006. Proceedings 2006 IEEE International Conference on. IEEE, 2006, pp. 2336-2341.

[4] R. F. Shepherd, F. Ilievski, W. Choi, S. A. Morin, A. A. Stokes, A. D. Mazzeo, X. Chen, M. Wang, and G. M. Whitesides, "Multigait soft robot," Proceedings of the National Academy of Sciences, vol. 108, no. 51, pp. 20 400-20403, 2011.

[5] N. Correll, C. D. Onal, H. Liang, E. Schoenfeld, and D. Rus, "Soft autonomous materials - using active elasticity and embedded distributed computation," in 12th Internatoinal Symposium on Experimental Robotics, New Delhi, India, 2010.

[6] C. D. Onal and D. Rus, "A modular approach to soft robots," in Biomedical Robotics and Biomechatronics (BioRob), 2012 4th IEEE RAS \& EMBS International Conference on. IEEE, 2012, pp. 10381045.

[7] R. J. Webster and B. A. Jones, "Design and kinematic modeling of constant curvature continuum robots: A review," The International Journal of Robotics Research, vol. 29, no. 13, pp. 1661-1683, 2010.

[8] M. W. Hannan and I. D. Walker, "Kinematics and the implementation of an elephant's trunk manipulator and other continuum style robots," Journal of Robotic Systems, vol. 20, no. 2, pp. 45-63, 2003.

[9] R. Cieslak and A. Morecki, "Elephant trunk type elastic manipulator-a tool for bulk and liquid materials transportation," Robotica, vol. 17, no. 1, pp. 11-16, 1999.

[10] R. Buckingham, "Snake arm robots," Industrial Robot: An International Journal, vol. 29, no. 3, pp. 242-245, 2002.
[11] I. A. Gravagne and I. D. Walker, "Uniform regulation of a multisection continuum manipulator," in Robotics and Automation, 2002. Proceedings. ICRA'O2. IEEE International Conference on, vol. 2. IEEE, 2002, pp. 1519-1524.

[12] W. McMahan, B. A. Jones, and I. D. Walker, "Design and implementation of a multi-section continuum robot: Air-octor," in Intelligent Robots and Systems, 2005.(IROS 2005). 2005 IEEE/RSJ International Conference on. IEEE, 2005, pp. 2578-2585.

[13] D. B. Camarillo, C. R. Carlson, and J. K. Salisbury, "Configuration tracking for continuum manipulators with coupled tendon drive," Robotics, IEEE Transactions on, vol. 25, no. 4, pp. 798-808, 2009.

[14] H. Wang, W. Chen, X. Yu, T. Deng, X. Wang, and R. Pfeifer, "Visual servo control of cable-driven soft robotic manipulator," in Intelligent Robots and Systems (IROS), 2013 IEEE/RSJ International Conference on, Nov 2013, pp. 57-62.

[15] M. B. Pritts and C. D. Rahn, "Design of an artificial muscle continuum robot," in Robotics and Automation, 2004. Proceedings. ICRA'04. 2004 IEEE International Conference on, vol. 5. IEEE, 2004, pp. $4742-4746$.

[16] R. Kang, D. T. Branson, T. Zheng, E. Guglielmino, and D. G. Caldwell, "Design, modeling and control of a pneumatically actuated manipulator inspired by biological continuum structures," Bioinspiration \& biomimetics, vol. 8, no. 3, p. 036008, 2013.

[17] C. D. Onal, X. Chen, G. M. Whitesides, and D. Rus, "Soft mobile robots with on-board chemical pressure generation," in International Symposium on Robotics Research (ISRR), 2011.

[18] A. D. Marchese, C. D. Onal, and D. Rus, "Soft robot actuators using energy-efficient valves controlled by electropermanent magnets," in Intelligent Robots and Systems (IROS), 2011 IEEE/RSJ International Conference on. IEEE, 2011, pp. 756-761.

[19] C. D. Onal and D. Rus, "Autonomous undulatory serpentine locomotion utilizing body dynamics of a fluidic soft robot," Bioinspiration \& biomimetics, vol. 8, no. 2, p. 026003, 2013.

[20] R. F. Shepherd, A. A. Stokes, J. Freake, J. Barber, P. W. Snyder, A. D. Mazzeo, L. Cademartiri, S. A. Morin, and G. M. Whitesides, "Using explosions to power a soft robot," Angewandte Chemie, vol. 125, no. 10, pp. 2964-2968, 2013.

[21] R. V. Martinez, J. L. Branch, C. R. Fish, L. Jin, R. F. Shepherd, R. Nunes, Z. Suo, and G. M. Whitesides, "Robotic tentacles with three-dimensional mobility based on flexible elastomers," Advanced Materials, vol. 25, no. 2, pp. 205-212, 2013.

[22] Y. Lianzhi, L. Yuesheng, H. Zhongying, and C. Jian, "Electropneumatic pressure servo-control for a miniature robot with rubber actuator," in Digital Manufacturing and Automation (ICDMA), 2010 International Conference on, vol. 1, 2010, pp. $631-634$

[23] M. Calisti, A. Arienti, M. Giannaccini, M. Follador, M. Giorelli, M. Cianchetti, B. Mazzolai, C. Laschi, and P. Dario, "Study and fabrication of bioinspired octopus arm mockups tested on a multipurpose platform," in Biomedical Robotics and Biomechatronics (BioRob), 2010 3rd IEEE RAS and EMBS International Conference on. IEEE, 2010, pp. 461-466.

[24] C. Laschi, M. Cianchetti, B. Mazzolai, L. Margheri, M. Follador, and P. Dario, "Soft robot arm inspired by the octopus," Advanced Robotics, vol. 26, no. 7, pp. 709-727, 2012.

[25] M. Calisti, M. Giorelli, G. Levy, B. Mazzolai, B. Hochner, C. Laschi, and P. Dario, "An octopus-bioinspired solution to movement and manipulation for soft robots," Bioinspiration \& biomimetics, vol. 6 , no. 3, p. 036002,2011

[26] T. Umedachi, V. Vikas, and B. Trimmer, "Highly deformable 3-d printed soft robot generating inching and crawling locomotions with variable friction legs," in Intelligent Robots and Systems (IROS), 2013 IEEE/RSJ International Conference on, Nov 2013, pp. 4590-4595.

[27] B. Jones and I. Walker, "Kinematics for multisection continuum robots," Robotics, IEEE Transactions on, vol. 22, no. 1, pp. 43-55, 2006

[28] A. Balestrino, G. De Maria, and L. Sciavicco, "Robust control of robotic manipulators," in Proceedings of the 9th IFAC World Congress, vol. 5, 1984, pp. 2435-2440.

[29] W. A. Wolovich and H. Elliott, "A computational technique for inverse kinematics," in Decision and Control, 1984. The 23rd IEEE Conference on, vol. 23. IEEE, 1984, pp. 1359-1363. 